\title{
THE STUDY OF DIRECT VIRTUAL PATH LAYOUT IN IP SWITCH NETWORKS ${ }^{\dagger}$
}

\author{
Jean-Lien C. Wu, Chien-Chung Chen and Jen-Kai Chen \\ Department of Electronic Engineering \\ National Taiwan University of Science and Technology \\ 43, Keelung Road, Section 4, Taipei, Taiwan, R.O.C. 106 \\ TEL: (886)-(2)-27376373 FAX: (886)-(2)-27376424 \\ Email: jcw@nlhyper.et.ntust.edu.tw
}

\begin{abstract}
The problem of the direct virtual path (VP) layout in IP Switch network is studied in this paper. With directVP layout, both the node forwarding cost and the transmission delay can be efficiently reduced in an IP Switch network. The equivalent bandwidth method is used to represent the resource requirement of the VPs and to simplify the problem of the VP management. Two VP layout policies, the periodic layout and the dynamic layout are discussed. With respect to the periodic directVP layout, an approximated optimal solution for forwarding cost reduction with both the maximumweight-first (MWF) selection and the clustering selection (CS) are provided to show that the forwarding cost can be reduced dramatically. Besides the forwarding cost reduction, the periodic layout also balances the load of physical links. A heuristic algorithm with the least-loaded routing (LLR) concept is proposed for dynamic layout to adjust the usage of VPs dynamically.
\end{abstract}

\section{INTRODUCTION}

The IP Switch technology [1-4] was first proposed by Ipsilon Networks Inc. to combine Internet protocol (IP) with ATM hardware, thus provides high speed switching capability. Using soft-state in the ATM hardware to cache the IP forwarding decision enables further traffic on the same IP flow to be switched by the ATM hardware rather than being forwarded by the IP routing software. The IP Switch is capable of distinguishing packet streams to be forwarded or switched.

Within an IP Switch network, direct links between all adjacent switch nodes form the set of default VPs as shown in Fig. 1, when the first packet of a flow from the source host within the domain of IP Switch A (IPSA) arrives at IPSA, the switch controller finds the route for this packet using the traditional routing algorithm like open-short-path-first (OSPF), and sends it out via the default channel to the next-hop node, i.e. IPSB in this scenario. With similar hop-by-hop forwarding procedure, this packet is transferred through IPSB, IPSC, IPSD, and then to the destination host within the IPSD domain. In this example, the forwarding cost of this packet is 4 , that is denoted by the number of switches along the

${ }^{\dagger}$ This research was supported by Computer and Communication Research Laboratories of Industrial Technology Research Institute of the Republic of China under grant G4-87026-a. forwarding route. In an IP Switch network, there exist mechanisms to differentiate a flow as forwarding flow or cut-through flow. The procedure to forward a flow has already been explained in Fig. 1. Once a cut-through flow from IPSA to IPSD is recognized, dedicated labels (VPI/VCI pairs) will be assigned for the links along the flow path. The cut-through function is implemented as label swapping, i.e. the switch updates the VPI and VCI fields in the cell header and then switches it to the output link right away. The switch performing VPI/VCI label swapping is called a VC-switch node. If there is a VP established between IPSA and IPSD, indicted as the "Direct-VP", IPSD becomes the immediate "next hop" of IPS-A and the packets from IPSA to IPSD can be passed via this direct-VP, the access costs and delay caused by the intermediate hops will then be reduced. In the directVP layout, IPSB and IPSC are VP-switch nodes which perform VP swapping so that the VPI/VCI label allocation will be handled at the terminating nodes of the direct-VP. Therefore, the forwarding cost for this packet reduced from 4 to 2 .

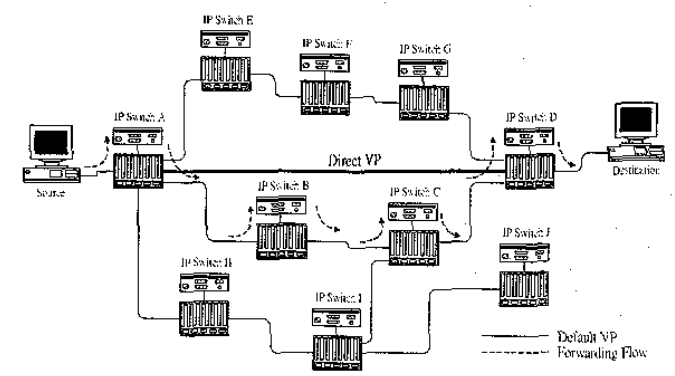

Figure 1: The usage of VP in an IP Switch Network

The key problem is to provide some efficient mechanisms to reconfigure and manage the VP layout in an IP Switch network. In the rest of this paper, two VP layout policies, the periodic layout and dynamic layout are discussed. Section 2 provides the formulation of the direct-VP layout problem. Periodic layout is described in section 3 and is executed periodically to obtain an approximated optimal direct-VP layout. The dynamic layout is used according to the physical traffic conditions to reallocate the VPs and is summarized in section 4. Section 5 presents the numerical results. Section 6 concludes the paper. 


\section{THE DIRECT-VP LAYOUT PROBLEM}

The major design issues of the direct-VP layout are described below:

1). The route of each direct-VP is obtained using the shortest path algorithm. To reduce the forwarding cost significantly, the number of hops along the route of each selected direct-VP has to be as high as possible with an upper bound to limit the maximum delay.

2). The traffic in every VP is a cost variable in the selection of VPs.

3). The total number of VPs in an IP Switch network is limited.

4). The route of each VP should be chosen so as to minimize the maximum load in the physical links.

5 ). There exists limitation in the physical link capacity.

The VP layout problem is an NP-complete subproblem [6][7], therefore we develop an approximation algorithm with provable performance guarantees and low complexity. To formulate the direct-VP layout problem, we first describe the following notations are used:

$\alpha$ : accessing cost for cell forwarding

$\beta$ : accessing cost for cell cut-through

$\delta \beta$ : accessing cost for direct-VP switching, $\delta<1$

$N_{k}$ : cost of network node, $k=1,2, \ldots, K$.

$P^{k}:$ the set of VPs connected to node $k$

$T P^{k}$ : the set of VPs where node $k$ is a transit hop

$B_{q}^{k}$ : the equivalent bandwidth of virtual path $q$,

$$
q \in p^{k}
$$

$h$ : the ratio of forwarded cells to total traffic

$l-h$ : the ratio of cut-through cells to total traffic

Without direct-VP layout, the processing cost of switch node $N_{k}$ is defined as

$$
N_{k}=\sum_{q \in P^{k}}\left[\alpha h B_{q}^{k}+\beta(1-h) B_{q}^{k}\right],
$$

and the total processing cost is $\sum_{k=1}^{K} N_{k}$. Considering direct-VP layout, the processing cost of a switch node $k$ becomes

$$
N_{k}=\sum_{q \in P^{k}}\left[\alpha h B_{q}^{k}+\beta(1-h) B_{q}^{k}\right]+\sum_{q \in T P^{k}}\left(\delta \beta B_{q}^{k}\right) .
$$

The direct-VP layout problem can be stated as the following:

Given a network configuration, the traffic load, and the maximum number of allowable VPs, find the optimum direct-VP layout such that the maximum link load is minimized such that the total processing cost of the network, $\sum_{k=1}^{K} N_{k}$, is minimized.

We proced the problem with the following two stages:

(1) The selection of the source-destination pairs for each direct-VP such that the total forwarding cost of the network is minimized.

(2) To compute the optimal routes of all direct-VPs so that the maximum link load is minimized, i.e. the link load has to be balanced extremely.

The total processing cost will decrease if the number of direct-VP increases because the processing cost of direct-VP switching is smaller than the cost of VC switching, i.e. $\beta>\delta \beta$, and it is much smaller than the cost of cell forwarding, i.e. $\alpha \gg \beta$.

An equivalent bandwidth of a set of VCs multiplexed onto a VP is defined as the amount of bandwidth required to achieve a desired QoS. In order to characterize the equivalent bandwidth or effective bit rate of VCs in terms of given parameters, the calculation of the equivalent bandwidth for VPs is based on their statistical characteristics at cell level using the fluid flow approximation and the stationary approximation methods $[8,9]$. We now briefly describe the concept of equivalent bandwidth. Suppose that the VCs within a VP have identical traffic characteristics and is modeled by a twostate Markov source where the peak rate is $R$, the mean arrival rate is $\lambda$ and the mean service rate is $\mu$. With respect to each VP, assume the buffer size is $G$, and the allowable cell loss probability is $\gamma$. The equivalent bandwidth at cell level using the fluid flow approximation for $K$ multiplexed VCs is denoted as $E B_{f}$ and can be expressed as

$E B_{f}=\frac{K\left\{\lambda+\mu-\ln (1 / \gamma) R / G-\sqrt{(\lambda+\mu-\ln (1 / \gamma) R / G)^{2}+4 \lambda R \ln (1 / \gamma) / G}\right\}}{-2 \ln (1 / \gamma) / G}$.

For $K$ multiplexed VCs, the mean aggregated bit rate is denoted by $A_{K}$, the standard deviation of the aggregated bit rate is $\eta_{K}$. The equivalent bandwidth using the stationary bit rate approximation, $E B_{s}$, can be written as

$$
E B_{s}=A_{K}+\sqrt{-2 \ln (\gamma)-\ln (2 \pi)} \eta_{K} .
$$

Based on the two approximations, we obtain the equivalent bandwidth, $E B$, as a function of the number of multiplexed VCs by the expression

$$
E B=\min \left\{E B_{f}, E B_{s}\right\} .
$$

For each VP to satisfy both the constraint of cell loss probability guaranteed QoS, we apply the equivalent bandwidth concept to determine the required bandwidths of VPs, thus the complicated VP bandwidth problem can be converted into a simple circuit-switching bandwidth problem. In an IP Switch network, the controller calculates the equivalent bandwidth for each VP connected to itself by the scheme explained above. The amount of equivalent bandwidth for each VP will be sent to the VP layout controller for the VPs layout and VP 
route decision.

\section{PERIODIC LAYOUT}

The objective in this section is to decide the overall direct-VP layout in an IP Switch network. The proposed approach in this paper for periodic layout decomposes the layout problem into two: the selection of a sourcedestination pair where a VP can be established and the routing for each VP. For the problems of VP layout, how to select the appropriate source-destination (SD) pairs and base on what criteria, is the most important consideration. The selection of VPs is used for forwarding cost reduction, a good selecting policy will reduce the cost dramatically. The routing of selected VPs for link load balance to avoid some physical links carry most traffic in a network. In the source-destination selection, two heuristic selecting policies are developed here; one is the maximum-weight-first (MWF) selection and the other is the clustering selection (CS).

For the MWF method, let the weight of an SD pair selection be the product of the traffic and the transit hop number along the corresponding VP that is the shortest path between the SD pair. The principle of the MWF selection is to select the VP with the maximum traffichop product among all. For example, if only one VP can be constructed in a network, the best efficiency is achieved by selecting the SD pair that has the maximum traffic-hop product so that the forwarding cost reduction is minimized. Let $S$ be the set of all source-destination pairs $S_{i j}$ in an IP Switch network, i.e. $S_{i j} \in S$. Each $S_{i j}$ is associated with an equivalent bandwidth of $\lambda_{i j}$. The transit hop number along $S_{i j}$ is denoted by hop $p_{i j}$. The selection of an SD pair is based on both weight (i.e. forwarding cost) defined as $W_{i j}=h o p_{i j} * \lambda_{i j}$. The selection criterion is to choose an SD pair $S_{i j}$ with the maximum weight from the set of $S$, then $S=S-S_{i j}$ and repeat the selection operation until either the number of selected VP reaches $n$ or there is no more sourcedestination pairs left in S. Let $P$ denote the selected $n$ SD pairs from $S$, then, the total reduction in total forwarding cost is given by

$$
\sum_{s_{i j} \in P} h o p_{i j} * \lambda_{i j}
$$

The MWF selection algorithm is a greedy scheme that results in a heuristic solution to the SD-pair selection problem.

Next we apply the clustering concept to the VP selection, this application was first applied by Chlamtac et al [6] to the VP layout problem in ATM networks. The principle is to select the next SD pair that is the farthest from the set of previously chosen cluster centers. It is based on the heuristic manner of that the farthest SD pair will result in maximal forwarding cost reduction because the farthest nodes can be served by this VP.

The clustering selection algorithm is described as follows. Parameter $S$ is defined as in the MWF selection for a set of all SD pairs, if we select a VP from $S$ which connects source node $i$ to destination node $j$, name this VP $P_{i j}$, we then say the node-pair $S D_{i j}$ is in the cluster with center $P_{i j}$. The cost of all SD pairs in the cluster with center $P_{\mathrm{ij}}$ is defined as the traffic-hop product as in the MWF selection.

$$
\operatorname{Cost}\left(S D_{x y}, P_{i j}\right)=\lambda_{x y}\left(h o p_{x i}+h o p_{i j}+h o p_{i j}\right),
$$

where hop $\mathrm{p}_{\mathrm{ij}}$ is $1, \mathrm{SD}_{\mathrm{xy}}$ is the $\mathrm{SD}$ pair that connects node $\mathrm{x}$ and node $\mathrm{y}, \lambda_{x y}$ is the equivalent bandwidth which is the same as in the MWF selection. Let $S_{c}$ be the subset of $S$, which consists of the VPs that are chosen. In [6] more than one SD pair will use the cluster center (VP) to construct their transmission path. Here we assume that only one SD pair in every cluster in order to apply the clustering policy to direct-VP problem in IP Switch network. Because the direct-VP is selected according to the traffic and hop number between the selected SD pair, and our goal is to provide a cost-saving path for the selected SD pair. The algorithm is provided in the following.

\section{Clustering Selection algorithm}

1). Find $V P_{1}$ from $S, V P_{1}$ is chosen arbitrarily, $S_{c}=\left\{V P_{1}\right\}$.

2). Repeat (3) to (5) until the number of VP selected is $n$.

3). For all $S D \in S-S_{C}$, find the minimum cost of every $\mathrm{SD}$ in $\operatorname{Cost}\left(S D, V P_{d}\right), V P_{d} \in S_{C}$.

4). Find the SD with maximum cost calculated in step (3), $S D \in S-S_{C}$, denote the SD of the maximum cost as

$V_{P_{\max }}$ and the VP number constraint in source and destination node of VPmax can not be violated.

5). $S_{C}=S_{C} \cup\left\{V P_{\max }\right\}$

The objective of VP routing is to find the set of optimal routes $P=\left\{P_{1}, P_{2}, . ., P_{n}\right\}$, where $P_{k}$ connects source node $S_{k}$ to destination node $D_{k}, k=1,2, \ldots, n$. The routing for each VP is also an NP-complete problem [7]. A near optimal concept is that if the maximum physical link load is the smallest possible, the maximum physical link load in an IP Switch network is minimum, then the set of VP routes connecting the SD pairs is called optimal. The routing algorithm described below is based on the proposals by Chlamtac et al [6][7]. This algorithm is proven that there exists a good bound on the deviation of the maximum link overload ratio from the best possible one. It is a near optimal algorithm with acceptable executing time and easy to be implemented. Two types of VP capacity demands were considered in [7], one is homogeneous demand, the same bandwidth requirement for each VP, the other is heterogeneous demand, a random bandwidth requirement is chosen independently for each VP. For comparison purposes, the optimum was computed by exhaustive search. Denoting by $R P$ as the maximum link load obtained by using the RPR algorithm and by $O P T$ as the true optimum value found by 
exhaustive search, the following results are obtained.

- In the homogeneous case, for $97 \%$ of all tests $R P=O P T$, in the remaining $3 \% R P<1.5 O P T$.

- In the heterogeneous case, for $33 \%$ of all tests $R P=O P T, 62 \%$ of the tests $O P T<R P<1.2 O P T$, and in the remaining $5 \%, 1.2 O P T<R P<1.5 O P T$.

The results show that for the homogeneous case the proposed algorithm performs excellently. For the heterogeneous case the performances is slightly worse, but it is still very good for the majority of cases. The main principles of the RPR algorithm are : 1) A shortest path route is selected to connect each $S D$ pair. 2) The weight for each physical link used by shortest path algorithm is assigned at random when VP routing is performed. 3) Routing of $P_{k}$ is made at random from the set of all possible shortest paths connecting $S_{k}$ to $D_{k}$. The random choice in 3 ) is that it tends to result in a more balanced use of network resources. The algorithm of route selection between the source and destination pairs is stated in the following steps:

Path Routing Algorithm

1). Set $i=1$.

2). Perform a random permutation of the numbers $1,2, \ldots, \mathrm{m}$.

3). Assign the random permutation numbers with the weight $w\left(l_{j}\right)$ to $l_{j}, \mathrm{j}=1,2, . ., \mathrm{m}$.

4). Find the inimum-weight path $P_{k}$ between the $S_{k}$ and $D_{k} . P_{k}$ is chosen as the VP connecting the $\left(S_{k}, D_{k}\right)$.

5). If $i=m$ then STOP else $i=i+1$ and go to Step 2 .

\section{DYNAMIC LAYOUT}

In the periodic layout policy, direct-VPs are selected based on the statistical traffic does not reflect the actual load, moreover, the Internet traffic is hardly to be predictable. When a network is operated after a period of time, periodic VP layout may not meet the real traffic requirement and may even violate the constraint of the physical link capacity. In an IP Switch network with best effort transmission policy, different VP layout policies may cause different loading of the physical link, an overloaded link will delay the transfer of cells and can't guarantee the quality of service. One needs to dynamically reconfigure a direct-VP to prevent unexpected conditions to overload the links. In the dynamic layout policy, we will focus on those links with traffic exceeding the predefined threshold value, and will try to re-allocate some direct-VPs with overloaded links to reduce the blocking probability of new arrivals. The following notations are used to explain the dynamic algorithm.

$P=\left\{P_{1}, P_{2}, ., P_{n}\right\}:$ the set of direct-VPs, $n$ is the number of direct-VPs in the network.

$\lambda=\left\{\lambda_{\mathrm{j}}, \lambda_{2}, \ldots, \lambda_{n}\right\}:$ the corresponding traffic of the direct-VPs.

$\mathrm{C}_{\mathrm{i}}$ : the capacity threshold of physical link $\mathrm{i}$, $\mathrm{i}=1,2, \ldots, \mathrm{m}, \mathrm{m}$ is the total number of physical links.

$R_{\mathrm{i}}$ : the set of VPs that use physical link $\mathrm{i}$

$\lambda_{R i}:$ total traffic of $\mathrm{R}_{\mathrm{i}}$

$S_{o}$ : the set of links whose traffic exceeds the threshold

The algorithm of the dynamic VP layout is described as follows:

1). For $S_{i} \in S_{o}$ repeat step 2 to step 7

2). $\varepsilon_{R i}=\lambda_{R_{i}}-C_{i}, \varepsilon_{R_{i}}$ is the amount of overload of link $\mathrm{i}$.

3). Repeat step 4 to step 7 when $\varepsilon_{R j}>\varepsilon, \varepsilon$ is the tolerance.

4). If there exists a $P_{j} \in R_{j}$ such that $\left|\lambda_{j}-\varepsilon_{R i}\right|$ is minimum and $P_{j}$ can be rerouted to another shortest path then go to step 5 , else go to step 6 .

5). Reroute the VP route to the new path and modify the traffic variables along $P_{j}, \varepsilon_{R i}=\lambda_{j}-\varepsilon_{R i}, \lambda_{R i}=\lambda_{R i}-\lambda_{j}$.

6). If $\lambda_{R i}>C$ then go to step 7 else go to step 1 .

7). New arrivals in $R_{i}$ will be blocked, go to step 1.

In the dynamic layout, there may be more than one path selected for the shortest-path route decision, the least-loaded routing (LLR) policy [11] is used here. The LLR policy has been demonstrated to be a simple and yet efficient algorithm for routing telephone calls. Because the equivalent bandwidth concept is applied, when a request arrives, the switch will only have to compare the equivalent bandwidth required from the new request with the free capacity of a direct-VP to decide whether to route this request through this direct-VP or not. The VP routing problem can, therefore, be transformed into an equivalent circuit-switched network routing problem. If no valid $P_{j}$ can be found among the overloaded links, then further arrivals in any VP belonging to $R_{i}$ will be blocked and the new arrival will be routed by the default VP path via hop-by-hop transmission.

\section{SIMULATION RESULTS}

We now present numerical examples employing the proposed direct-VP layout algorithm with the network model shown in Fig. 2 which is an NSFNET T3 backbone network consisting of 12 nodes and 132 source-destination pairs [9]. Table 1 is the traffic load measured for all SD pairs in the NSFNET.

Assume every node shown in Fig. 2 is an IP Switch node, in this example network, there are 12 IP Switch nodes. If a fully connected network is considered, every node must have $11 \mathrm{VPs}$ to connect with each other, in other words, the out-degree number of every switch is 11 . To simplify the control of VP connection assignment and management, we assume the in-degree number is equal to the out-degree number for all switch nodes in our simulation. The elements of the traffic load matrix are assumed to be the equivalent bandwidth for every source-destination pair. We will pay more attention to the 
reduction in forwarding cost here, therefore, suppose the traffic load matrix in table 1 are equivalent bandwidth of forwarded traffic.

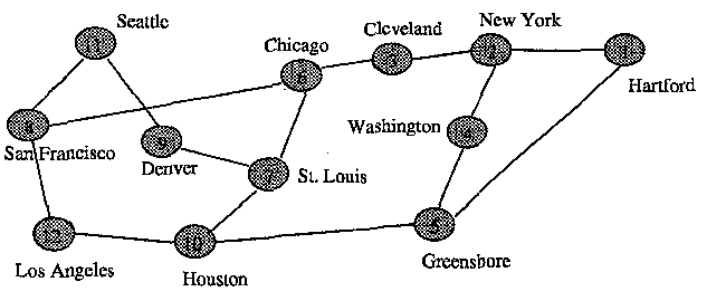

Figure 2: Network model (NSFNET)

Table.1 Offered traffic load matrix (NSFNET)

\begin{tabular}{|c|c|c|c|c|c|c|c|c|c|c|c|c|}
\hline & 1 & 2 & 3 & 4 & 5 & 6 & 7 & 8 & 9 & 10 & 11 & 12 \\
\hline 1 & & 209 & 13219 & 6079 & 3180 & 147 & 2252 & 8462 & 64 & 6158 & 57 & 3047 \\
\hline 2 & 150 & & 115 & 53 & 28 & 1 & 20 & 74 & 1 & 54 & 1 & 27 \\
\hline 3 & 12021 & 147 & & 4280 & 2239 & 103 & 1586 & 5958 & 45 & 4336 & 40 & 2146 \\
\hline 4 & 4836 & 59 & 3719 & & 901 & 42 & 638 & 2397 & 18 & 1744 & 16 & 863 \\
\hline 5 & 2400 & 29 & 1846 & 855 & & 21 & 317 & 1190 & 9 & 866 & 8 & 428 \\
\hline 6 & 105 & 1 & 81 & 37 & 20 & & 14 & 52 & 1 & 38 & 1 & 19 \\
\hline 7 & 1673 & 20 & 1287 & 596 & 312 & 14 & & 829 & 6 & 603 & 6 & 299 \\
\hline 8 & 7044 & 86 & 5417 & 2508 & 1312 & 61 & 929 & & 26 & 2541 & 24 & 1257 \\
\hline 9 & 46 & 1 & 35 & 16 & 9 & 1 & 6 & 23 & & 17 & 1 & 8 \\
\hline 10 & 4906 & 60 & 3773 & 1747 & 914 & 42 & 647 & 2432 & 18 & & 16 & 876 \\
\hline 11 & 41 & 1 & 31 & 15 & 8 & 1 & 5 & 20 & 1 & 15 & & 7 \\
\hline 12 & 2295 & 28 & 1765 & 817 & 427 & 20 & 303 & 1138 & 9 & 828 & 8 & \\
\hline
\end{tabular}

The impact of the in-degree and out-degree numbers on forwarding cost reduction is shown in Fig. 3. The major advantage employing direct-VP layout is that the number of forwarded packets for each switch node is reduced, so that both the access time and the transmission delay can be saved and, therefore, the performance can be improved. In the example shown in Fig. 2, suppose there exists a direct-VP from node 1 to node 6 , the transit hop count number is 2 , i.e. node 2 and node 3 , the traffic from node 1 to node 6 is 147 packets given in Table 1 . If there exists no direct-VP between node 1 and node 6 , node 2 and node 3 will have to access all these 147 forwarded packets, because nodes 2 and 3 belong to the shortest-path-node set from node 1 to node 6 . To explain the reduction in forwarding cost, we define the weight ratio as

weight ratio $=(\mathrm{A} / \mathrm{B}) * 100 \%$,

where $A$ is the overall forwarding cost for transit hops in an IP Switch network and B is the total forwarding cost in an IP Switch network without direct-VP layout.

Fig. 3 shows the relationship between the weight ratio and the number of direct-VP for each switch node. Fig. 4 shows that when the in-degree number (out-degree) is 2 , the weight ratio using the MWF selection is $56 \%$ and is about $57 \%$ when the clustering selection policy is applied. It shows quite an amount of improvement in forwarding cost reduction. The weight ratio is below $2 \%$ when the in-degree number is 7 no matter what selection policy is applied, in other words, in-degree $=7$ is good to be used as a direct-VP layout constraint in the example network for further simulations. A different perspective of the weight is the transmission delay for packets to go through the VP path. Suppose the packet processing time of an IP Switch node is 1 , then $h o p_{i j} * \lambda_{i j} * 1$ is the total transmission time of this VP if the physical link transmission delay is negligible. Therefore, Fig. 3 also presents the transmission time reduction ratio when the direct-VP layout is applied to an IP Switch network.

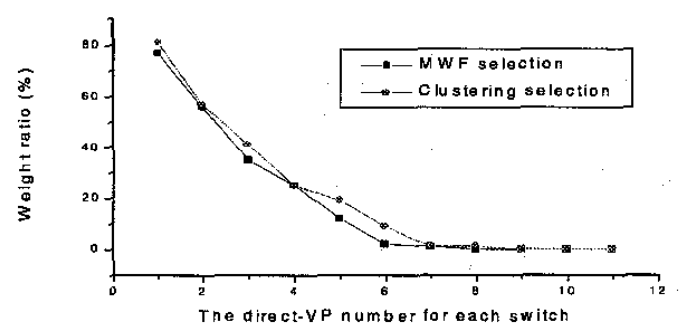

Figure 3: Forwarding cost ratio in a direct-VP layout network

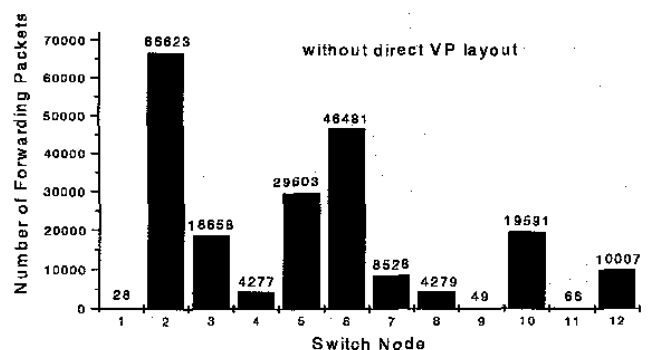

Figure 4: Forwarding packets for each IP Switch node without direct-VP layout.

From the simulation results shown in Fig. 3, each IP Switch node is allocated 7 VPs. Without the direct-VP layout, the number of packets forwarded and accessed by every switch node is shown in Fig. 4. The worst case is in node 2 where it must have the capability to forward 66623 packets. Results of the direct-VP layout network with the MWF selection is shown in Fig. 5. The maximum number of packets forwarded is 749 for switch node 3 , we can see the obvious improvement here, the number of packets forwarded by a switch node drops from 66623 packets to 749 packets. That is, the proposed direct-VP layout policy and algorithm can have up to $99 \%$ forwarding cost reducing.

Fig. 6 shows the load of every physical link, the maximum loaded link is physical link 5 without directVP layout and the load is 56203 . With direct-VP layout using the MWF selection, the maximum link load is reduced to 47515 , the reduction ratio is $84.5 \%$, the result of the direct-VP layout with clustering selection is 46003 , and the reduction ratio is $81.9 \%$. 


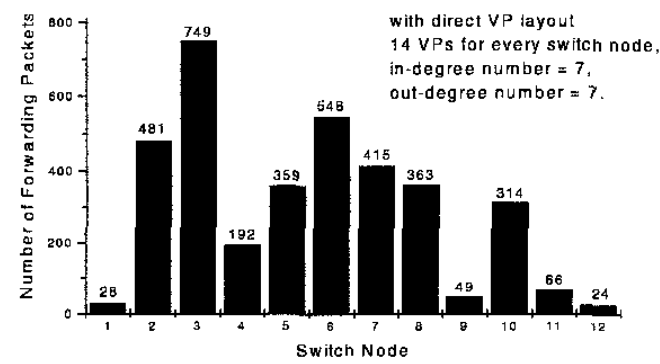

Figure 5: Forwarding packets for each IP Switch node with direct-VP layout

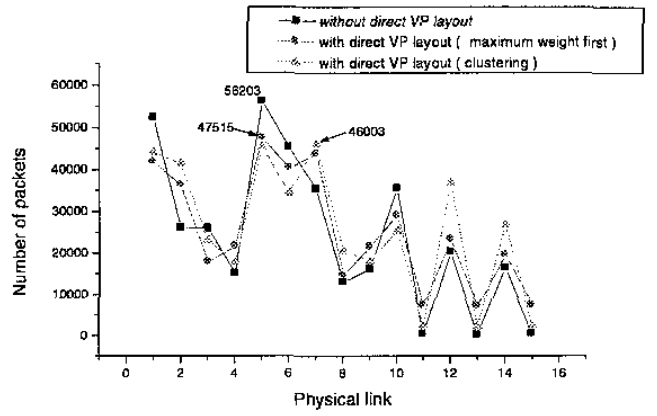

Figure 6: The usage of physical links

\section{CONCLUSION}

In this paper, we proposed the direct-VP layout algorithms for IP Switch networks to efficiently reduce the forwarding cost and transmission delay. We tried to minimize the forwarding cost of every switch node through the selection of VP and the route decision for every VP. With the numerical examples, we have demonstrated that this design method can achieve quite an amount of improvement. In addition to the forwarding cost reduction, the direct-VP layout algorithm can also balance the network load.

Two VP selection methods are compared, namely the maximum-weight-first (MWF) and the clustering selection, both can achieve the cost reduction efficiently. The MWF selection performs better in the forwarding cost reduction, in the example network, $43 \%$ forwarding cost and transmission delay reduction can be obtained by assigning for direct-VP to every switch node, two for indegree connections and two for out-degree connections. The clustering selection performs better in the link load balance, based on the simulation results, the maximum link load is reduced to $82 \%$ for the case that 7 direct-VP are applied. We also proposed an algorithm to dynamically adjust the VPs that experiences overload, the algorithm may not provide the optimal solution, but it is simple and easy to be implemented.

\section{REFERENCES}

[1] Newman, P., Lyon, T. and Minshall, G., "Flow Labelled IP:A Connectionless Approach to ATM,"
Proc. IEEE INFOCOM'96, San Francisco, CA, USA, pp.1251-1260, 1996

[2] Newman, P. , Edwards, W., Hinden, R., Hoffman, E., Liaw, F. C., Lyon, T. and Minshall, G., "Ipsilon Flow Management Protocol Specification for IPv4," IETF RFC 1953, May 1996

[3] Newman, P. , Edwards, W., Hinden, R., Hoffman, E., Liaw, F. C., Lyon, T. and Minshall, G., "Transmission of Flow Labelled IPv4 on ATM Data Links," IETF RFC 1954, May 1996

[4] Newman, P. , Edwards, W., Hinden, R., Hoffman, E., Liaw, F. C., Lyon, T. and Minshall, G., "Ipsilon General Switch Management Protocol Specification Version 1.1," IETF RFC 1987, Aug. 1996

[5] Burgin, J. and Dorman, D., "Broadband ISDN Resource Management: The Role of Virtual Paths," IEEE Commun. Magazine, 29, 1991

[6] Chlamtac, I., Farago, A. and Zhang, T., "How to Establish and Utilize Virtual Paths in ATM Networks," Proc. IEEE ICC'93, Geneva, Switzerland, pp.1368-1372, 1993

[7] Chlantac, I., Farago, A. and Zhang, T., "Optimizing the System of Virtual Paths," IEEE/ACM Trans. on Networking, Vol. 2, No. 6, pp.581-587, 1994

[8] Guerin, R., Ahmadi, H. and Naghshineh, M., "Equivalent Capacity and Its Application to Bandwidth Allocation in High-Speed Networks," IEEE J. Select. Areas in Commun., Vol.9, No. 7, pp.968-981, Sep.1991

[9] Ryu, B. H., Ohsaki, H., Murata, M. and Miyahara, H., "Design Algorithm for Virtual Path Based ATM Networks," IEICE Trans. Commun. Vol. E79-B, No. 2, pp.97-107, 1996

[10] Ko, T. M. and Chang, M., "Least Measured Cost Routing in VP-Based ATM Networks," Proc. IEEE GLOBECOM'97, Phoenix, AR, USA, pp.18201824,1997

[11] Kolarov, A. and Hui, J., "Least Cost Routing in Multiple-Service Networks," Proc. IEEE INFOCOM'95, Boston, Mass. USA, pp.1482-1489, 1995 